\title{
Effect of Flaxseed Extract on the Liver Histological Structure in Streptozotocin Induced Diabetic Rats
}

\author{
Imad M. Al-Ani ${ }^{a^{*}}$, Ahmed N. Abired ${ }^{a}$, Basma E. Mustafa ${ }^{b}$, Emad N. Abdel Wahab ${ }^{a}$, Marwan S. Azzubaidi ${ }^{c}$ \\ ${ }^{a}$ Department of Basic Medical Science, Kulliyyah of Medicine, International Islamic University Malaysia, \\ Kuantan, Pahang, MALAYSIA. \\ ${ }^{b}$ Department of Basic Medical Science, Kulliyyah of Dentistry, International Islamic University Malaysia, \\ Kuantan, Pahang, MALAYSIA. \\ 'Pharmacology Unit, Faculty of Medicine, Universiti Sultan Zainal Abidin, Kampus Perbatam, 20400 Kuala \\ Terengganu, MALAYSIA
}

\begin{abstract}
Introduction: Diabetes mellitus has become a serious warning to mankind health all over the world. The management goal of diabetes is to keep blood glucose levels as close as possible to healthy individuals. Medications used to treat diabetes are usually associated with complications and may cause different side effects. Many traditional anti-diabetic plants have become popular in the management of diabetes mellitus. Flaxseed has been used as traditional medicine for centuries. Objective: This study aimed to evaluate the hepatoprotective effects of flaxseed extract in streptozotocin (STZ)-induced diabetic rats. Methods: Diabetes mellitus was induced in Sprague-Dawley rats using a single injection of streptozotocin $(60 \mathrm{mg} / \mathrm{kg}$ i.p.). The rats were divided into five groups of 8 rats each. Group NC, normal control rats; Group NF, normal rats treated with flaxseed extract $(400 \mathrm{mg} / \mathrm{kg})$; Group DC, diabetic control rats; Group DG, diabetic rats treated with glibenclamide $(0.6 \mathrm{mg} / \mathrm{kg})$; Group DF, diabetic rats treated with flaxseed extract $(400 \mathrm{mg} / \mathrm{kg})$; for 4 weeks. Results: There were significant increase in relative liver weight, blood glucose levels in DC group comparing to NC group $(\mathrm{p}<0.05)$. The disturbance of these parameters was ameliorated in DF and DG groups. Histological observation revealed congestion of central veins, degeneration of hepatocytes, and reduced glycogen granules in DC group. These pathological changes were ameliorated in the flaxseed extract and glibenclamide treated rats. Conclusion: Flaxseed extract may represent a candidate alternative treatment to control diabetes mellitus and its related hepatopathy.
\end{abstract}

KEYWORDS: Diabetes mellitus, glibenclamide, flaxseed, liver, rats, streptozotocin

\section{INTRODUCTION}

Diabetes is one of the most common chronic diseases worldwide that affects individuals worldwide and is one of the major causes of death. Diabetes is a major threat to global public health, and the numbers of diabetic patients are rapidly increasing world-wide. ${ }^{1}$ In 2013, the international diabetes federation approximated that 382 million adults had diabetes and 5.1 million deaths occur annually due to diabetes; the prevalence of diabetes has doubled in the last three decades and is projected to continue rising to 592 million cases by $2035 .^{2}$ Diabetes is characterized by hyperglycaemia,

Corresponding author:

Imad M. AL-Ani.

Department of Basic Medical Sciences,

Kulliyyah of Medicine,

International Islamic University Malaysia.

Phone: 006017-9776014.

E-mail: imad_alani@yahoo.com glucose intolerance, insulin insensitivity or absolute absence of insulin due to damage of the pancreatic $B$-cells. ${ }^{3}$ This frequently results in severe metabolic imbalances and pathological changes in many tissues. $^{4}$

Diabetes mellitus is associated with a number of common symptoms, such as polyurea and polydipsia, and long term complications, including retinopathy, neuropathy, nephropathy, and atherosclerosis. Recent studies has reported that patients with diabetes have a high susceptibility for different liver diseases, including non-alcoholic fatty liver disease, abnormal liver enzymes, acute liver failure, cirrhosis, and hepatocellular carcinoma. $^{5}$

Experimental studies have demonstrated many histopathological changes in the liver; such as hepatocytic necrosis, vacuolations of the cytoplasm and the nucleus, fragmentation of hepatocytes and sinusoids, fatty degeneration and vascular congestion of the central vein. ${ }^{6,7}$ 
Several therapeutic agents have been developed to manage diabetes and its associated complications. However, the current conventional therapies may cause many side effects, the patients may develop resistance, and they do not adequately inhibit the development of the associated complications. ${ }^{8,9}$ This necessitates discovery of more potent therapies. Certain plant extracts have been used traditionally to manage diabetes and several more are under study for their potential in managing hyperglycaemia and its complications. The herbal therapies have potential to adequately manage the disease while eliminating the adverse effects associated with conventional therapies. ${ }^{10,11}$

Flaxseed is a crop that is grown worldwide due to its associated health benefits. It contains many biologically active components including ALA, fibre, protein and phenolic compounds such as lignanss. ${ }^{12}$ Linolenic acid is helpful in controlling the level of circulating bad cholesterol, and hence prevents the development of cardiac diseases. Flaxseed is one of the richest sources of lignanss, which have been studied extensively due to their therapeutic potential. ${ }^{13}$ Lignanss have been shown to prevent the development of various human cancers. ${ }^{14}$ Progress is being made in its use in the management of diabetes mellitus and its complications. The underlying mechanisms of the anti-diabetic effect of lignanss involve enhancing glycemic control, increasing the insulin sensitivity, and inhibition of hepatic gluconeogenesis. ${ }^{3,15}$ The present study has been designed to study the protective effect of flaxseed extract on the histological changes of liver in STZ- induced diabetic rats.

\section{MATERIALS AND METHODS}

\section{Flaxseed}

Flaxseeds were obtained from the Department of Botany, Faculty of Science; Philadelphia University, Jordan. Two hundred grams of the defatted powder was macerated with $1.2 \mathrm{~L}$ of $70 \%$ ethanol solvent for 24 hours at $30^{\circ} \mathrm{C}$. The extract then was filtered by a sand core funnel and concentrated at $40^{\circ} \mathrm{C}$ and 90 rpm using a rotary evaporator, followed by freeze drying for 4 days at $-80^{\circ} \mathrm{C}$. The obtained crude extract was diluted with distilled water to yield light yellow syrup which was administrated to the experimental animals at a dose of $400 \mathrm{mg} / \mathrm{kg}$ body weight.

\section{Animals and experimental design}

Forty healthy adult male Sprague Dawley rats aged from 10 to 12 weeks old (weighing 200-250g) were used in this study. The animals were housed in polypropylene cages under the standard laboratory conditions (adequate cross ventilation; temperature: $24 \pm 2{ }^{\circ} \mathrm{C}$; $12: 12$ hrs light: dark cycle; relative humidity: $46-79 \%$ ), and were allowed two weeks period to acclimatize prior to the experiment. The rats were maintained on standard commercial dry pellet diet and water ad libitum. The animals were randomly divided into five groups of 8 animals in each group as described in Table 1.

Table 1: Animal grouping

\begin{tabular}{|c|c|c|c|}
\hline No. & $\begin{array}{l}\text { Group } \\
\text { initial }\end{array}$ & Group definition & Treatment \\
\hline 1 & NC & Normal control rats & - Pellets and water \\
\hline 2 & NF & Normal rats treated with flaxseed & $\begin{array}{l}\text { - Pellets and water } \\
\text { - Flaxseed } 400 \mathrm{mg} / \mathrm{kg}\end{array}$ \\
\hline 3 & DC & Diabetic control rats & $\begin{array}{l}\text { - Pellets and water } \\
\text { - STZ 60mg/kg }\end{array}$ \\
\hline 4 & DG & $\begin{array}{l}\text { Diabetic rats treated with } \\
\text { glibenclamide }\end{array}$ & $\begin{array}{l}\text { - Pellets and water } \\
\text { - STZ 60mg } / \mathrm{kg} \\
\text { - Glibenclamide } 0.6 \mathrm{mg} / \mathrm{kg}\end{array}$ \\
\hline 5 & DF & Diabetic rats treated with flaxseed & $\begin{array}{l}\text { - Pellets and water } \\
\text { - STZ 60mg/kg } \\
\text { - Flaxseed } 400 \mathrm{mg} / \mathrm{kg}\end{array}$ \\
\hline
\end{tabular}


The rats were fasted overnight (12-14 hours). The animals were given a single intraperitoneal STZ injection of $60 \mathrm{mg} / \mathrm{kg}$ of body weight dissolved in citrate buffer $\mathrm{PH}$ 5.4. In order to prevent hypoglycemia, STZ-treated animals received a solution of $5 \%$ glucose instead of normal drinking water over 24 hours following the treatment. Normal control rats received an equivalent volume of distilled water. On the fifth day of postinduction, Accu-Chek Performa glucometer was used to measure the level of fasting blood glucose.

Only rats with high level of fasting blood glucose (more than $14 \mathrm{mmol} / \mathrm{L}$ ) were considered as diabetic rats. The sixth day of diabetes induction was considered as the first day of treatment. Syringe and special designed metal ball-ended needle were used to feed the rats daily by gavage. NF and DF groups received $400 \mathrm{mg} / \mathrm{kg}$ flaxseed extraction diluted with distilled water. DG group were fed with glibenclamide $0.6 \mathrm{mg} / \mathrm{kg}$, and the remaining animals (NC and DC groups) were untreated with flaxseed or glibenclamide.

The measurement of body weight (g) for all rats was recorded on the $1^{\text {st }}, 5^{\text {th }}, 10^{\text {th }}, 20^{\text {th }}$, and $30^{\text {th }}$ day of the experiment using Mettler Toledo SB16000 Balance (Painesville, OH, USA). At sacrifice, the measurement of liver relative to weight was determined for all rats according to the equation:

The relative percentage $=\frac{\text { liver weight }}{\text { body weight }} \times 100$
of liver weight

The study protocol was approved by the Institutional Animal Care and Use Committee (IACUC), International Islamic University Malaysia (IIUM). The experiment was conducted according to the Guidelines for the Care and Use of Laboratory Animals of the Kulliyyah of Medicine, IIUM.

\section{Histological Studies}

At the end of the experimental period, the tested animals were sacrificed using Ketamine as an aesthetic. Liver specimens were rapidly removed, fixed in $10 \%$ formal saline for 72 hours, dehydrated through graded alcohols and cleared using two changes of xylene and embedded in paraffin wax. Serial transverse sections of 4-5 micron thickness were prepared using the microtome, stained Haematoxylin and eosin ( $\mathrm{H} \& \mathrm{E})$, others stained by periodic Acid-Schiff stain (PAS) with salivary amylase application.

\section{Statistical analysis}

Data analyses were performed using statistic software IBM SPSS Statistics 21 (IBM Corporation, NY, USA). Data were expressed as mean \pm standard deviation (SD). The results were considered as significant at $P$ value less than 0.05 . Student $t$-test were used to compare the data within the group. Additionally, one way analysis of variance (ANOVA) was used to compare the data between experimental groups, followed by Tukey's post hoc test for multiple groups' comparison.

\section{RESULTS}

\section{Effects of flaxseed extract on body weight and liver weight}

In normal groups, NC and NF, the rats maintained a steady increase in body weight and reached to about $290 \pm 9.78 \mathrm{~g}$ on day 30 compared to $227 \pm$ $7.97 \mathrm{~g}$ on the first day of the study (Figure 1. A). No significant difference $(p>0.05)$ in body gained weight was observed between NC and NF groups during the whole study period. In DC group, the rats exhibited a slight increase of mean body weight from $227 \pm 7.94 \mathrm{~g}$ on day 1 to about $229 \pm 6.51 \mathrm{~g}$ on day 5 , followed by a progressive decrease to reach $196 \pm 12.04 \mathrm{~g}$ on day 30 . The reduction of mean body weight in DC group was significant when compared to NC group $(\mathrm{p}<0.05)$.

Treatment with glibenclamide and flaxseed extract seems to reduce the rate of weight loss that has been noticed in untreated STZ diabetic rats. The mean body weight in DG group progressively increased from $229 \pm 6.76 \mathrm{~g}$ on day 1 to $260 \pm 9.05$ $\mathrm{g}$ on day 30. While in DF group, it gradually decreased from $229 \pm 7.21 \mathrm{~g}$ on day 1 to $206 \pm 8.29$ $g$ on day 30. The ameliorative effect of glibenclamide on body weight was statistically significant compared to DC group $(p<0.05)$. However, the alteration of body weight in DF group didn't reach a significant level in comparison with DC group ( $p>0.05)$ (Figure 1. A).

The absolute liver weight was nearly similar in all groups. However, the relative weight of liver with respect to body weight was higher in diabetic groups than in normal groups (Table 2).

The relative weight of liver in both NC and NF groups was approximately $2.94 \pm 0.22 \mathrm{~g}$. DC group was presented with a relative liver weight of $4.40 \pm$ $0.28 \mathrm{~g}$, which is significantly higher than that in NC group $(p<0.05)$. The increase in liver relative weight in diabetic rats was ameliorated in DF and DG groups to be $4.31 \pm 0.45 \mathrm{~g}$ and $3.34 \pm 0.25 \mathrm{~g}$ respectively, the latter was significantly less than in DC group $(\mathrm{p}<0.05)$ (Table 2$)$. 

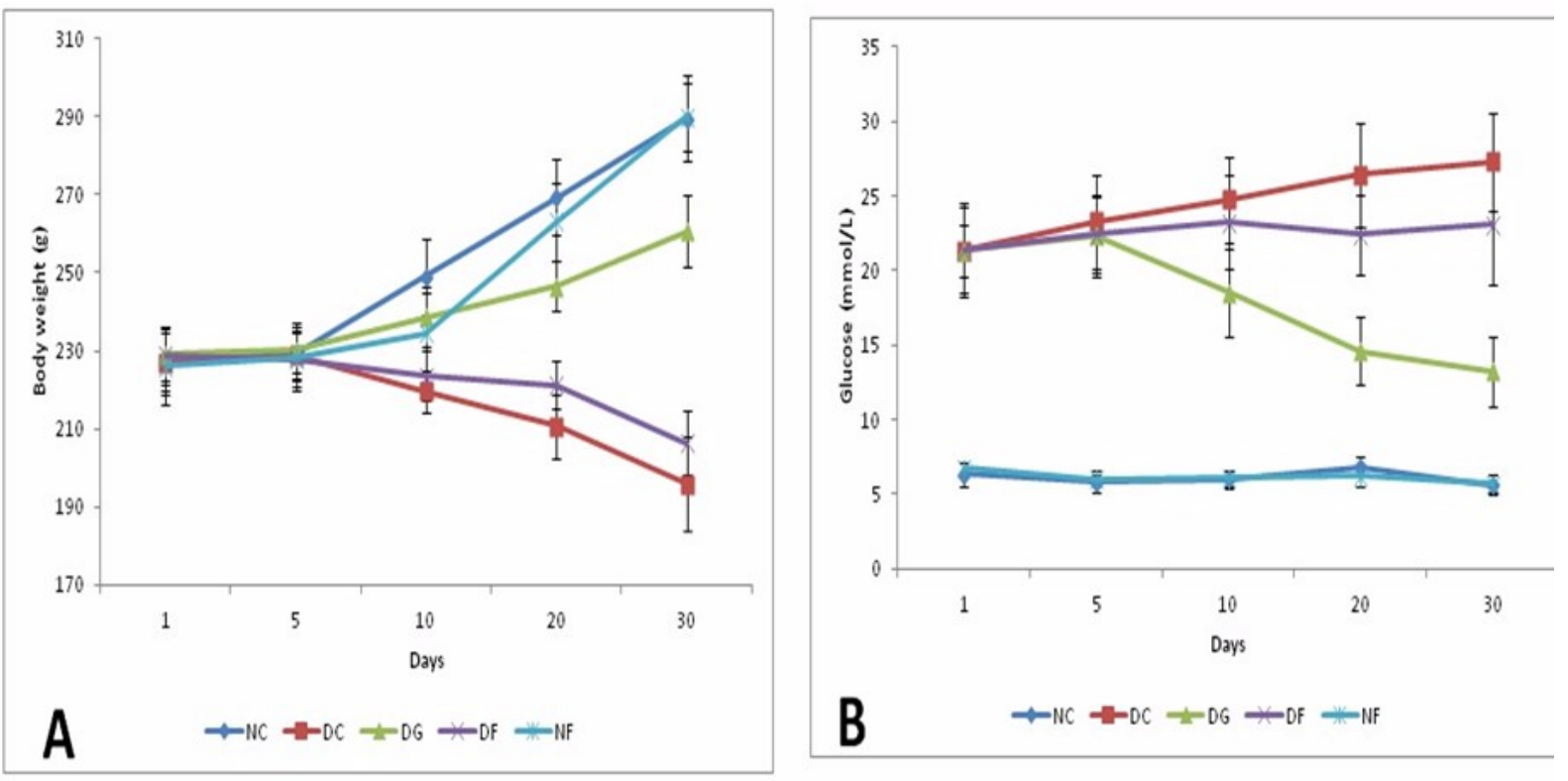

Figure 1. A. Mean \pm SD of body weight of all groups during different days. B. Mean \pm SD of fasting blood glucose level of all groups during different days.

Table 2 Mean body weight, absolute liver weight and relative liver weight of experimental groups*

\begin{tabular}{cccc}
\hline Group & Body & $\begin{array}{c}\text { Absolute liver } \\
\text { w }\end{array}$ & $\begin{array}{l}\text { Relative liver } \\
\text { eight w }\end{array}$ \\
\hline NC & $289.6 \pm$ & $8.50 \pm 0.41$ & $2.94 \pm 0.17$ \\
& $\begin{array}{c}10.89 \\
195.8 \pm\end{array}$ & $8.60 \pm 0.26$ & $4.40 \pm 0.28$ \\
DC & 12.04 & & \\
& & $8.69 \pm 0.63$ & $3.34 \pm 0.25$ \\
DG & $260.7 \pm 9.05$ & & \\
& & $8.88 \pm 0.65$ & $4.31 \pm 0.45$ \\
DF & $206.3 \pm 8.29$ & & \\
& & $8.50 \pm 0.59$ & $2.94 \pm 0.27$ \\
NF & $290.1 \pm 8.67$ & & \\
\hline
\end{tabular}

\section{Effects of flaxseed extract on fasting blood glucose level}

In normal groups, NC and NF, the rats have consistently maintained their fasting blood glucose levels close to $6 \pm 0.62 \mathrm{mmol} / \mathrm{L}$. On the other hand, all diabetic groups had much higher levels of fasting blood glucose following administration of STZ (Figure 1. B).

The rats in the diabetic groups DC, DG and DF, had an initial mean fasting blood glucose level of approximately $21 \pm 2.59 \mathrm{mmol} / \mathrm{L}$. Subsequently, in DC group, these levels were raised up to $27 \pm 3.30$ $\mathrm{mmol} / \mathrm{L}$ on day 30 . The elevation of fasting glucose level in DC group was statistically significant as compared to NC group $(p<0.05)$ (Figure 1. B).

Treatment of diabetic rats with glibenclamide and flaxseed extract has reduced the elevated levels of fasting blood glucose throughout the study period. In DG group, the mean fasting blood glucose slightly increased to $22 \pm 2.74 \mathrm{mmol} / \mathrm{L}$ on day 5 , then dropped to $18 \pm 2.92 \mathrm{mmol} / \mathrm{L}$ on day 10 , and continuously decreased to reach $15 \pm 2.29$ $\mathrm{mmol} / \mathrm{L}$ and $13 \pm 2.37 \mathrm{mmol} / \mathrm{L}$ on days 20 and 30 , respectively. The reduction of fasting glucose level in DG group was statistically significant as compared to $D C$ group $(p<0.05)$.

In DF group, the rats showed a slight elevation in the mean fasting glucose level up to $23 \pm 3.14$ $\mathrm{mmol} / \mathrm{L}$ on the $10^{\text {th }}$ day of study period, followed by a stable glucose level until the end of the experiment. However, the hypoglycemic activity of flaxseed extract didn't reach to a significant level compared to the DC group ( $p>0.05)$ (Figure 1. B).

\section{Effects of flaxseed extract on liver histopathological changes}

Liver sections obtained from normal groups, NC and $\mathrm{NF}$, revealed normal histological architecture; with regular hepatic lobules with central veins and peripheral portal areas were observed. Moreover, regular distinct hepatocytes with sinusoidal spaces were extending radially from the central veins to the boundaries of portal areas.

The sinusoids were lined by endothelial cells containing Kupffer cells (Figure 2, A \&C). PAS stained sections of NC and NF groups showed abundant distribution of glycogen granules within the cytoplasm of hepatocytes (Figure 2, B \&D). 

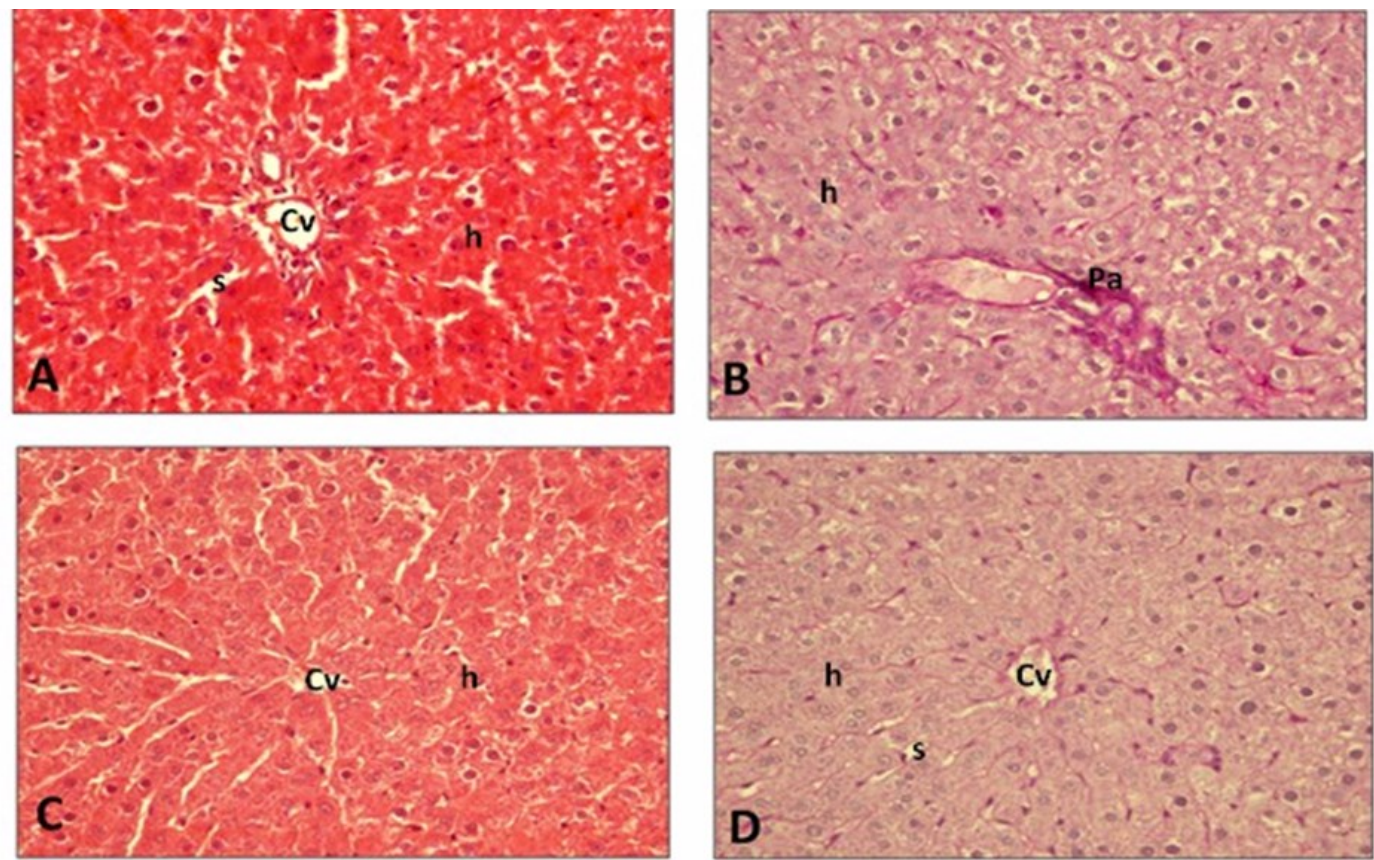

Figure 2. Liver sections of: A. normal control rats showing normal histological structure, regular distinct hepatocytes with sinusoidal spaces arranged radially around the central vein. B. normal control rats with normal histological structure and numerous glycogen granules distributed throughout the slide area and portal area. C. normal rats treated with flaxseed extract illustrating normal histological structure of hepatocyte, blood sinusoid. D. normal rats treated with flaxseed extract showing normal histological structure and PAS positivity granules in most of the hepatocytes. "CVv, central vein; $h$, hepatocyte; Pa, portal area; s, sinusoid”. (A and C, H\&E; B and D, PAS, original magnification X40).

In diabetic (DC) group, liver sections stained with H\&E and PAS showed noticeable pathological changes as compared to normal control group. These changes were indicated by the presence of congested central veins, degenerated and irregularshaped hepatocytes, cloudy swelling and vacuolization of cytoplasm (Figure $3, \quad A$ \& B). Additionally, the hepatocytes of diabetic untreated rats were further presented with a marked reduction of glycogen granules (Figure 3, B).
However, treatment with flaxseed extract and glibenclamide has reduced the severity of these changes. Sections obtained from DF and DG groups showed normal hepatocytes with no signs of cytoplasmic cloudy swelling or vacuolization, less congestion of central veins, and normal contents of glycogen granules, indicating a restoration of normal liver architecture (Figure 3, C \& D) (Figure 4. $A \& B)$.
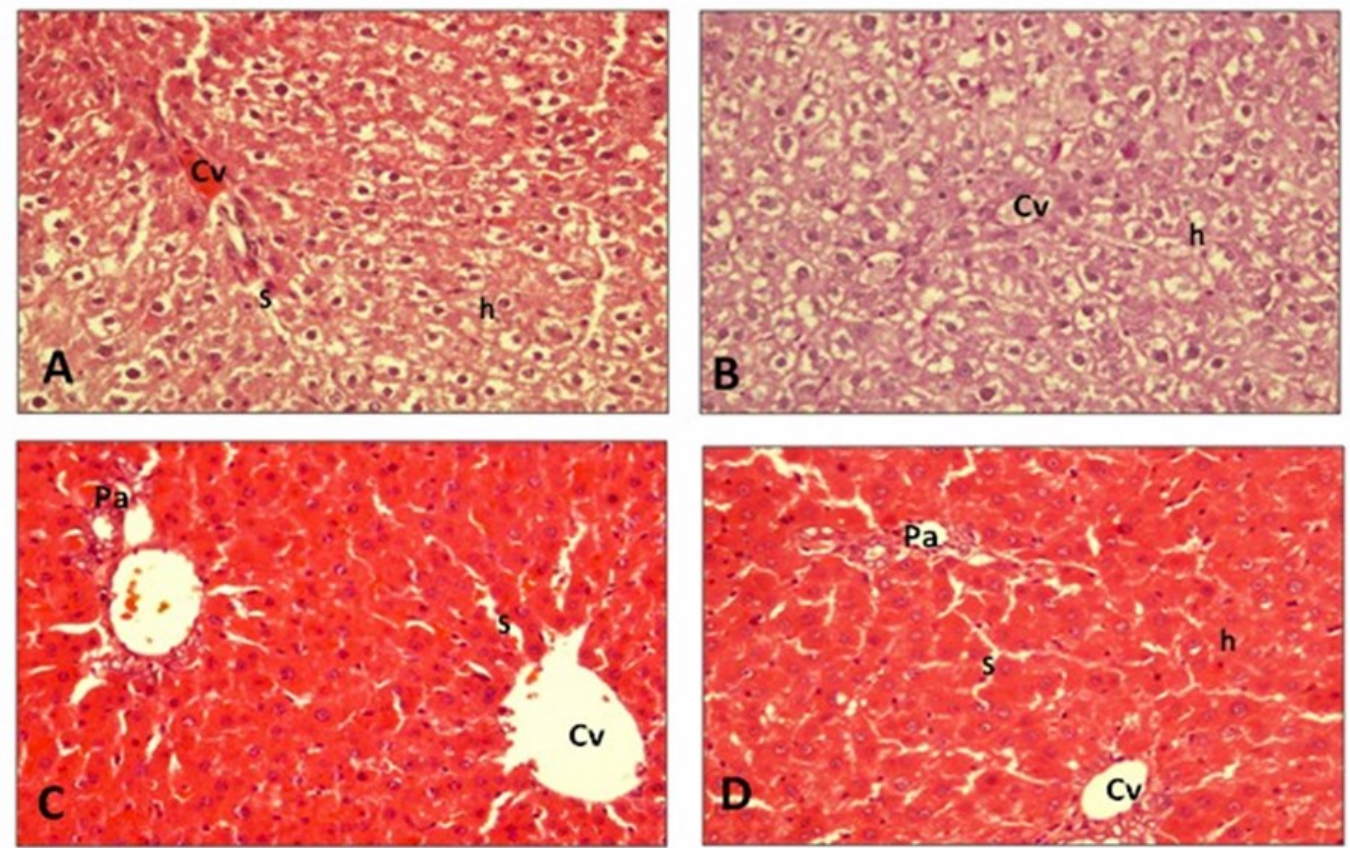

Figure 3. Liver sections of: A \& B. STZ diabetic rats showing degeneration of hepatocytes, vacuolization of cytoplasm, and cloudy swelling, congested central vein and loss of glycogen granule in B. H\&E, X400. C. diabetic liver rats treated with flaxseed extract presented with less pathological changes and improved liver architecture. H\&E, X400. D. diabetic rats treated with glibenclamide demonstrating restoration of the normal liver structure. "Cv, central vein; $\mathrm{h}$, hepatocyte; Pa, portal area; s, sinusoid”. (A, C\& D, H\&E; B, PAS, original magnification X40). 

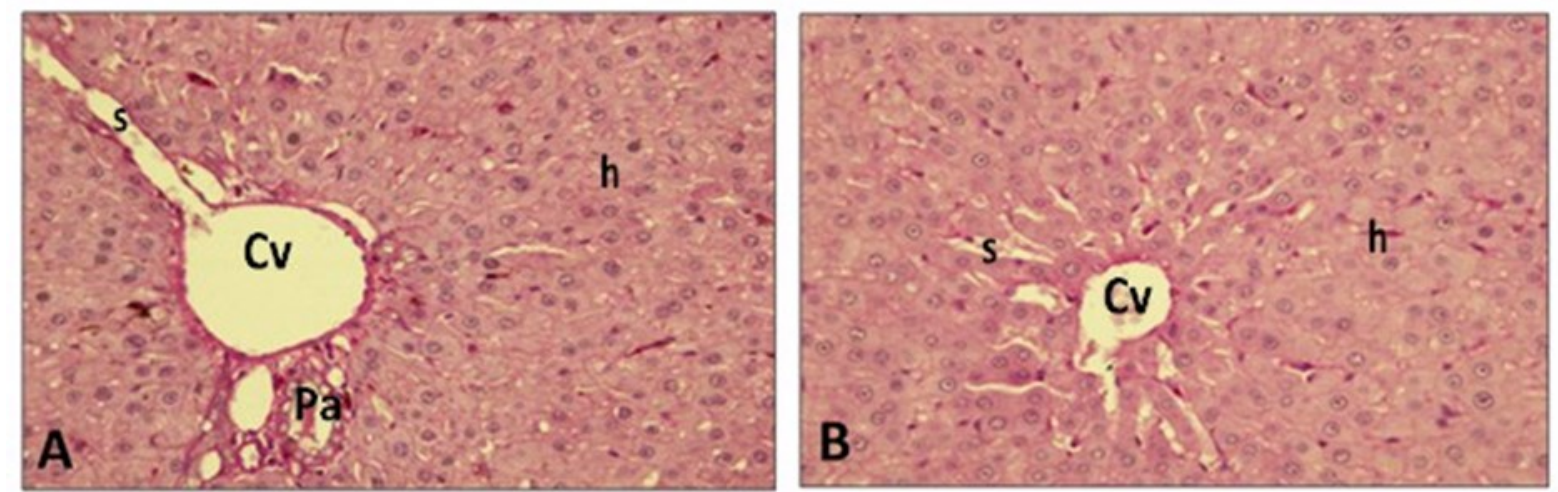

Figure 4. Liver sections of diabetic rats treated with (A) flaxseed extract and (B) glibenclamide showing restoration of liver architecture and normal glycogen granule contents in the hepatocytes. "Cv, central vein; h, hepatocyte; Pa, portal area; s, sinusoid". (PAS, original magnification X40).

\section{DISCUSSION}

Diabetes mellitus is one of the most common causes of liver damage (Manna et al., 2010). It has been correlated with the entire spectrum of liver diseases, including abnormal levels of liver enzymes, non-alcoholic fatty liver disease and liver cirrhosis and carcinoma. ${ }^{16}$ There is clear evidence that oxidative stress during diabetes plays an important role in the induction of these diseases (Adeyemi et al., 2014). The conventional treatment of diabetes mainly involves a sustained reduction of blood glucose levels using different agents, such as sulfonylurea and thiazolidinediones. ${ }^{17}$ However, these synthetic drugs do not adequately inhibit the development of the associated complications, and furthermore, they may cause a variety of side effects. ${ }^{18}$ Hence, there is a demand for alternative therapies to manage diabetes mellitus and its associated complications. ${ }^{17}$

Certain herbal extracts have shown the ability to treat diabetes and prevent the development of its long-term complications without causing adverse effects. Flaxseed is a rich source of lignans, which has antidiabetic and antioxidant properties. ${ }^{19,20}$ Considering these properties, this study was conducted to evaluate the hepatoprotective effect of flaxseed extract in a model of STZ-diabetic rats. Changes in different parameters, including body weight, liver relative weight, blood glucose level and histopathological picture were determined in the diabetic rats treated with flaxseed extract, and compared with those receiving a commercially available drug, glibenclamide.

The present study showed a significant increase of fasting blood glucose levels in the untreated diabetic rats. Oral administration of flaxseed extract reduced the levels of fasting blood glucose in diabetic rats; which is consistent with earlier reports that demonstrated antidiabetic activity of flaxseed and attributed it to its content of lignanss that inhibit the hepatic gluconeogenesis., ${ }^{3,15}$ Treatment with glibenclamide has also decreased the blood glucose levels in diabetic rats, and it was found to be more effective in comparing with flaxseed extract.

In the present study, STZ-induced diabetic rats showed severe loss of body weight. This reduction is due to the degradation of structural proteins during diabetes mellitus. ${ }^{21,22}$ However, there is an increase in liver weight in proportion to the body weight. Liver hypertrophy during diabetes mellitus might be the result of an increased hepatic accumulation of triglycerides. ${ }^{21}$ These findings are in agreement with the previous studies which reported increased liver relative weight in STZ-induced diabetic rat. ${ }^{6,21,23}$ Treatment with glibenclamide and to a lesser extent flaxseed extract has reversed the progressive changes of body weight and liver weight in diabetic rats. The capability of these treatments to protect the change of body weight and liver weight seems to be due to their ability to reduce hyperglycemia.

Evaluation of liver sections obtained from STZdiabetic rats revealed the presence of several histopathological changes including congestion of central veins, degeneration of hepatocytes, reduction of glycogen granules, swelling and vacuolization of cytoplasm. Our findings are in good agreement with several previous studies, which reported similar histopathological changes following induction of diabetes using STZ injection. ${ }^{6,24,25}$ However, less pathological changes and improved liver architecture were observed in flaxseed extract and glibenclamide treated diabetic rats, indicating protective effects of these treatments against the hepatic changes associated with diabetes.

The mechanism of the ameliorative effect of flaxseed extract on the histopathological changes in STZ-induced diabetic rats is still not clear. However, flaxseed is one of the richest sources of the plantbased $\underline{w}-3$ fatty acid, alpha-linolenic acid (ALA), and its ingestion may help in preventing or treating a variety of diabetic complications. ${ }^{26}$ Dietary intake of $1.25 \pm 0.07 \mathrm{~g}$ ALA among adults' diabetic patients with peripheral neuropathy reduced the odds of 
peripheral neuropathy. ${ }^{27}$ Experimental study in Zucker diabetic fatty Gmi-fa/fa female rats showed that Secoisolariciresinol diglucoside (SDG) antioxidant from flaxseed can prevent the development of type 1 diabetes by approximately $71 \%$ (90) and type 2 diabetes by $80 \% .{ }^{28}$ Significant improvements were observed in glycemic control in type 2 diabetic patients treated for 12 weeks with lignin supplementation derived from flaxseed. ${ }^{15}$ Furthermore, lignans act as antioxidants through the direct scavenging of radicals and by preventing lipid peroxidation. ${ }^{3}$ Further studies are needed to investigate the effects of ALA, lignin and antioxidants from flaxseed on liver morphology in experimental diabetic animals.

\section{CONCLUSION}

It is concluded that daily treatment with flaxseed extract improves blood glucose level, and histopathological status of STZ-induced diabetes mellitus. The experimental evidence obtained from this study indicates that flaxseed extract represents a candidate alternative treatment to control diabetes mellitus and its related hepatopathy

\section{Acknowledgement}

This study was supported by the grant program from RMC, International Islamic University Malaysia; Research Endowment Fund Grant No. (EDW B 14214-1099).

\section{Conflict of interest statement}

The authors declare that they have no personal or financial conflict of interest.

\section{REFERENCES}

1. Ramudu SK, Korivi M, Kesireddy N, et al. Nephro-Protective Effects of a Ginger Extraction Cytosolic and Mitochondrial Enzymes against Streptozotocin (STZ)-Induced Diabetic Complications in Rats. Chinese J Physiol 2011; 54: 79-86.

2. Guariguata L, Whiting DR, Hambleton I, et al. Global estimates of diabetes prevalence for 2013 and projections for 2035. Diabetes research and clinical practice 2014; 103: 137 49.

3. Prasad K. Suppression of phosphoenolpyruvate carboxykinase gene expression by secoisolariciresinol diglucoside (SDG), a new antidiabetic agent. International J Angiology 2002; 11: 107-9.

4. Ansari MH, Karimipour M, Salami S, et al. The Effect of Ginger (Zingiber officinale) on Oxidative Stress Status in the Small Intestine of Diabetic Rats. Int J Endocrinol Metab 2008; 3: 144-50.

5. Abolfathi AK, Mohajeri D, Rezaie A, et al. Protective Effects of Green Tea Extract against Hepatic Tissue Injury in Streptozotocin-Induced
Diabetic Rats. Evidence-Based Complementary and Alternative Medicine 2012; Volume 2012, Article ID 740671, 10 pages doi:10.1155/2012/740671

6. Al-Ani IM, Al-Mishadani NS, Muslih RK, et al. Histological liver changes in streptozotocin induced diabetic mice. IMJM 2009; 8: 1-3.

7. Kumar MSJ. Hepatoprotective and antidiabetic effect of aqueous extract of cynodon dactylon in streptozotocin induced diabetic rats histological study. Internat J Pharma Bio Sciences 2015; 6: 627-35.

8. Zia T, Hasnain SN, Hasan SK. Evaluation of the oral hypoglycaemic effect of Trigonella foenum-graecum L. (methi) in normal mice. J Ethnopharmacol 2001; 75: 191-5.

9. Halim EM. Lowering of blood sugar by water extract of Azadirachta indica and Abroma augusta in diabetes rats. Ind J exper biol 2003; 41: 636-40.

10. Kesari AN, Kesari S, Singh SK, et al. Studies on the glycemic and lipidemic effect of Murraya koenigii in experimental animals. $\mathrm{J}$ Ethnopharmacol 2007; 112: 305-11.

11. Modak M1, Dixit P, Londhe J, et al. Indian Herbs and Herbal Drugs Used for the Treatment of Diabetes. J Clin Biochem Nutrit 2007; 40: 163-73.

12. Ganorkar P, Jain RK. Flaxseed - a nutritional punch. International Food Res J 2013; 20: 51925.

13. Sicilia T, Niemeyer HB, Honig DM, et al. Identification and stereochemical characterization of lignanss in flaxseed and pumpkin seeds. J Agric Food Chem. 3003; 51: 1181-8.

14. Trentin GA, Moody J, Torous DK, et al. The influence of dietary flaxseed and other grains, fruits and vegetables on the frequency of spontaneous chromosomal damage in mice. Mutation research 2004; 551: 213-22.

15. Pan A, Sun J, Chen Y, et al. Effects of a flaxseed-derived lignin supplement in type 2 diabetic patients: A randomized, double-blind derived lignin supplements in type 2 diabetic patients: A randomized, double-blind, crossover trial. PLoS ONE 2007; 2: e1-148.

16. Tolman KG, Fonseca V, Dalpiaz A, et al. Spectrum of liver disease in type 2 diabetes and management of patients with diabetes and liver disease. Diabetes care 2007; 30: 734-43.

17. Gengiah K, Hari R, Anbu J. Antidiabetic antihyperlipidemic and hepato-protective effect of Gluconorm-5: A polyherbal formulation in steptozotocin induced hyperglycemic rats. Ancient Sci Life 2014; 34: 23-32.

18. Noor A, Gunasekaran S, Soosai MA, et al. Antidiabetic activity of Aloe vera and histology of organs in streptozotocin-induced diabetic rats. Current science 2008; 94: 1070-6.

19. Hu C, Yuan YV, Kitts, et al. Antioxidant activities of the flaxseed lignans secoisolariciresinol diglucoside, its aglycone 
secoisolariciresinol and the mammalian lignanss enterodiol and enterolactone in vitro. Food and chemical toxicology 2007; 45: 221927.

20. Lowcock EC, Cotterchio M, Boucher BA. Consumption of flaxseed, a rich source of lignanss, is associated with reduced breast cancer risk. Cancer causes and control 2013; 24: 813-6.

21. Zafar M, Naqvi SN. Effects of STZ-Induced Diabetes on the Relative Weights of Kidney, Liver and Pancreas in Albino Rats: A Comparative Study. Int J Morphol 2010; 28: 135-42.

22. Cheng D, Daye; Liang, et al. Antihyperglycemic effect of Ginkgo biloba extract in streptozotocin-induced diabetes in rats. BioMed Research International 2013; Volume (2013), Article ID 162724, 7 pages http://dx.doi.org/10.1155/2013/162724

23. Adaramoye OA. Antidiabetic effect of kolaviron, a biflavonoid complex isolated from Garcinia kola seeds, in Wistar rats. African health sciences 2012; 12: 498-506.

24. Mahmoud MF, Sakr SM. Hepatoprotective Effect of Bee Propolis in Rat Model of StreptozotocinInduced Diabetic Hepatotoxicity: Light and Electron Microscopic Study. Life Science J 2013; 10: 2048- 54.

25. Motshakeri M, Ebrahimi M, Goh YM, et al. Effects of Brown Seaweed (Sargassum polycystum) Extracts on Kidney, Liver, and Pancreas of Type 2 Dsiabetic Rat Model. Evidence-based complementary and alternative medicine 2014; Article ID 379407, 11 pages http:/ /dx.doi.org/10.1155/2014/379407

26. Rodriguez-Leyva D, Bassett CM, McCullough R, et al. The cardiovascular effects of flaxseed and its omega-3 fatty acid, alpha-linolenic acid. Can J Cardiol 2010; 26: 489-96.

27. Tao M, McDowell MA, Saydah SH, et al. Relationship of polyunsaturated fatty acid intake to peripheral neuropathy among adults with diabetes in the National Health and Nutrition Examination Survey (NHANES) 1999. 2004. Diabetes Care 2008; 31: 93-5.

28. Prasad K. Secoisolariciresinol diglucoside from flaxseed delays the development of type 2 diabetes in Zucker rat. J Lab Clin Med 2001; 138: $32-9$ 